\title{
Comparison of functional fibrinogen assessment using thromboelastography with the standard von Clauss method
}

\author{
Ivo Fluger ${ }^{\mathrm{a}}$, Katerina Maderovaa ${ }^{\mathrm{a}}$, Martin Simek ${ }^{\mathrm{a}}$, Roman Hajek ${ }^{\mathrm{a}}$, Jana Zapletalova ${ }^{\mathrm{b}}$, Vladimir Lonsky
}

\begin{abstract}
Aim. To compare assessment of fibrinogen by thromboelastography with the standard von Clauss method.
Methods. Observational prospective study.

Results. Thromboelastography provides direct and complex evaluation of the entire coagulation cascade based upon changes in blood viscosity. It affects both platelets and plasma components. New application of this method measures fibrinogen contribution to coagulation as opposed to fibrinogen antigen levels measured by immunoassay. Paired samples from 117 patients before cardiopulmonary bypass were compared. A moderate correlation was found between fibrinogen and functional fibrinogen with a Spearman correlation coefficient of 0.476 .
\end{abstract}

Conclusion. The functional fibrinogen test is a valid point-of-care method for fibrinogen assay with a moderate correlation to the standard method.

Key words: fibrinogen, functional fibrinogen, thromboelastography, coagulation, cardiopulmonary bypass

Received: February 3, 2011; Accepted: June 1, 2011; Available online: June 28, 2011

hppt://dx.doi.org/10.5507/bp.2011.035

${ }^{a}$ Department of Cardiac Surgery, University Hospital Olomouc and Faculty of Medicine and Dentistry, Palacky University Olomouc, Czech Republic

${ }^{b}$ Department of Biomedicine and Statistics, Faculty of Medicine and Dentistry, Palacky University Olomouc Corresponding author: Ivo Fluger, e-mail: flugeri@fnol.cz

\section{INTRODUCTION}

Fibrinogen concentrations are traditionally measured using clottable protein methods, end point-detection techniques or immunochemical tests. The most commonly used fibrinogen assay relies on the von Clauss method. This method involves a 10 -fold dilution of plasma, which ensures that fibrinogen is the rate-limiting step in clot formation. Subsequently, an excess of thrombin is added to the sample and the time to clot formation is measured. The clotting time is inversely related to fibrinogen concentration. The Thrombelastograph (TEG; Haemoscope Corp, IL, USA) measures the clot's viscosity using a cup and pin assembly. A blood sample $(360 \mu \mathrm{l})$ is placed in a plastic cup into which a pin is suspended. The cup, heated to $37^{\circ} \mathrm{C}$, oscillates through an arc of 4 degrees and $45 \mathrm{~min}$. The pin is attached to a torsion wire which is monitored for motion. When the blood is fully liquid, movement of the cup does not affect the pin. However, as a clot begins to form, the viscosity of the blood increases and the pin becomes coupled to the motion of the cup and the torsion wire to generate an electric signal. After online computerized processing it forms a characteristic tracing curve. This thromboelastographic curve includes both plasma and platelet components of the coagulation. Reaction time (R) is the time from start of the reaction until a measurable clot is detected. $\mathrm{K}$ time is from the $\mathrm{R}$ point until a certain clot firmness is achieved. The angle $(\alpha)$ reflects the rate of clot formation. The maximum amplitude (MA) of clot shear elasticity reflects the contribution of fibrin and platelets to clot strength. The MA may be converted by the formula: $\mathrm{Gf}=5000 \times \mathrm{MA} /$
$(100-\mathrm{MA})$ to a shear elasticity value (Gf) in dynes per square centimeter.

The principle of the new modification of thromboelastography is as follows: The functional fibrinogen reagent (lyophilized tissue factor with platelet inhibitor- Gp IIb/ IIIa receptor blocker) fully inhibits the platelets, excluding their contribution to clot strength (MA) and therefore measures only the fibrinogen contribution to clot strength. The value MA of a platelet-free plasma clot is proportionate to the functional fibrinogen concentration. Analytical software calculates the functional fibrinogen level (MAFF or FLEV) through the transformation of the MA value.

\section{MATERIAL AND METHODS}

The study comprised 117 elective patients, operated for ischemic heart disease with planned CPB. Included were only patients without known coagulopathy or hepatopathy and with antiplatelet drugs discontinued more than one week before surgery. The study was approved by the local ethics committee. Blood samples were obtained from the central vein cannula after $10 \mathrm{ml}$ blood removal. Fibrinogen levels by the von Clauss method and also thromboelastography were assessed. Additionally, functional fibrinogen was determined by modified TEG (ref. ${ }^{1}$ ). The method is based on determining the fibrinogen portion of the thromboelastography curve after inhibition of the platelet portion, as described above. Routine quality testing was performed as recommended by the TEG manufacturer. Data were statistically processed using the 


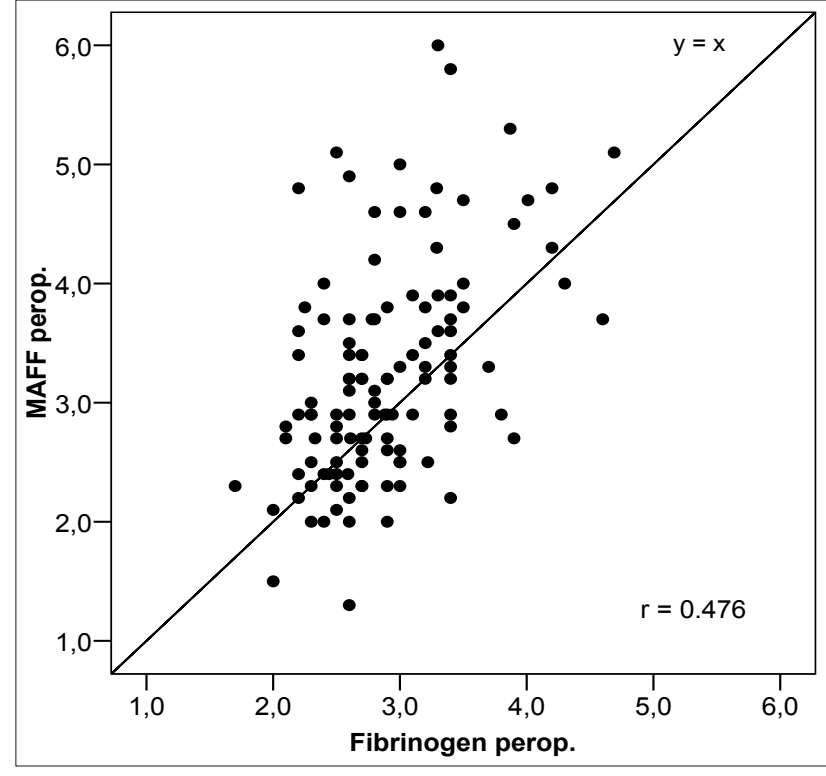

Fig. 1. Correlations between fibrinogen and functional fibrinogen.

SPSS Statistics 15 software (SPSS Inc., Chicago, USA). Correlation coefficients were calculated using Spearman's correlation to assess the relationships between fibrinogen and functional fibrinogen levels.

\section{RESULTS}

Blood samples from 117 patients were assessed to determine the relationship of functional fibrinogen and fibrinogen levels obtained by the von Clauss method. (Fig. 1) presents the linear regression of functional fibrinogen (MAFF) to fibrinogen. There was a moderate correlation $(r=0.476, p<0.0001)$ between fibrinogen and functional fibrinogen.

\section{DISCUSSION}

Our results indicate that functional fibrinogen levels (MAFF) obtained by thromboelastography are proportionate to fibrinogen levels obtained by the standard method. The correlation is not as good as that reported by Carroll et al. ${ }^{1}$ who had fewer patients in the study. Unlike their patients, the subjects in this study were not healthy. Thus, their fibrinogen may have had a functional deficiency analogous to that in a study by Miller et al. ${ }^{2}$ and Deptula et $\mathrm{al}^{3}$.

The von Clauss method relies on detection of the actual clot in excess of thrombin. It may be affected by fibrin degradation products, polymerization inhibitors as other inhibitors of fibrin formation ${ }^{4}$. This analysis is possible only with small concentrations of heparin, which is a serious limitation in cardiac surgery. Unlike the von Clauss method, the thromboelastographic function fibrinogen assay is possible even with full heparinization, e.g. when

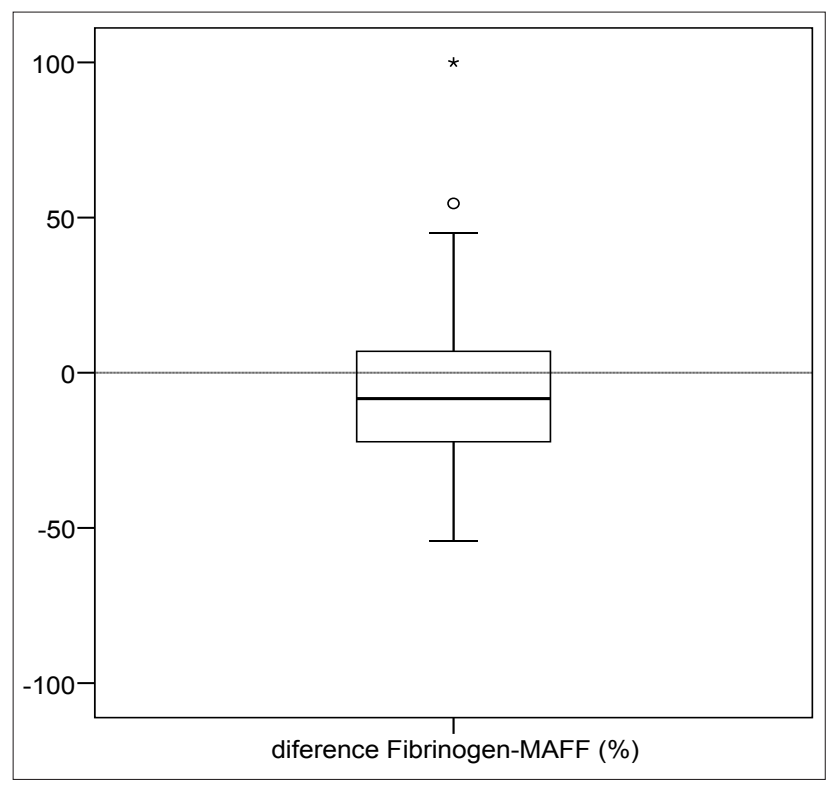

Fig. 2. The box plot shows a distribution of differences between fibrinogen and functional fibrinogen in $\%$.

on cardiopulmonary bypass. Another advantage of pointof-care TEG is its rapidity ${ }^{5}$ and ability to measure fibrinolysis ${ }^{6}$.

\section{CONCLUSION}

Functional fibrinogen test is a point-of-care method for fibrinogen assay with a moderate correlation to the standard method.

\section{ACKNOWLEDGEMENT}

The study is supported by the Czech Ministry of Health project NS9614-3/2008.

\section{REFERENCES}

1. Carroll RC, Craft RM, Chavez J, Snider CC. Kirby R, Cohen E. Measurement of functional fibrinogen levels using the Thrombelastograph J Clinic Anesth 2008;20:186-90.

2. Miller BE, Tosone SR, Guzzetta NA, Miller JL, Brosius KK. Fibrinogen in Children Undergoing Cardiac Surgery: Is It Effective? Anesth Analg 2004;99(5):1341-6.

3. Deptula J, Glogowski K, Merrigan K, Hanson K, Felix D, Hammel J, Duncan K Evaluation of biocompatible cardiopulmonary bypass circuit use during pediatric open heart surgery. J Extra Corpor Technol 2006;38(1):22-6.

4. Koh SC, Chew CY, Viegas OA, Choo M, Ratnam S Influence of circulating D-dimer levels on assays of fibrinogen. Ann Acad Med Singapore 1994;23(6):856-60.

5. Spalding GJ, Hartrumpf M, Sierig T, Oesberg N, Kirschke CG, Albes JM Bedside thrombelastography. Cost reduction in cardiac surgery. Anaesthesist 2007;56(8):765-71.

6. Hajek R, Nemec P, Bruk V, Fluger I, Jarkovsky J, Nemethova D. Hypercoagulation during cardiac surgery with cardiopulmonary bypass detected with thromboelastography. \Applied Cardiopulm Pathophys 2007;11(2):80. 